Relations industrielles

Industrial Relations

\title{
Kent Rowley, The Organizer, A Canadian Union Life, by Rick Salutin, Toronto, James Lorimer, 1980, 163 pp.
}

\section{F. J.K. Griezic}

Volume 36, numéro 3, 1981

URI : https://id.erudit.org/iderudit/029199ar

DOI : https://doi.org/10.7202/029199ar

Aller au sommaire du numéro

Éditeur(s)

Département des relations industrielles de l'Université Laval

ISSN

0034-379X (imprimé)

1703-8138 (numérique)

Découvrir la revue

Citer ce compte rendu

Griezic, F. J. (1981). Compte rendu de [Kent Rowley, The Organizer, A Canadian Union Life, by Rick Salutin, Toronto, James Lorimer, 1980, 163 pp.] Relations industrielles / Industrial Relations, 36(3), 704-705.

https://doi.org/10.7202/029199ar

Tous droits réservés @ C Département des relations industrielles de l'Université Laval, 1981
Ce document est protégé par la loi sur le droit d'auteur. L'utilisation des services d'Érudit (y compris la reproduction) est assujettie à sa politique d'utilisation que vous pouvez consulter en ligne.

https://apropos.erudit.org/fr/usagers/politique-dutilisation/ 
tissage, ainsi que le rôle de l'aspect affectif dans l'explication des choix individuels menant à un comportement donné.

Dans le chapitre 2 ("The Theory", pp. 23 à 65) les auteurs passent à une description proprement dite de la théorie. On retrouve à la page 24 une représentation schématique de la théorie où les variables introduites dans le modèle sont nommées et placées en ordre séquentiel d'influence causale. Dans les chapitres 3 et 4 (pp. 65 à 114), la «mécanique» du jugement humain est disséquée, et les auteurs s'appliquent ensuite à démontrer comment le comportement individuel est finalement le résultat d'une série de jugements ou d'évaluations faites par les individus concernés. Finalement, les chapitres 5 à 8 inclusivement sont destinés à illustrer comment la théorie permet de renouveler la compréhension que nous pouvons avoir des thèmes traditionnels que sont la théorie des rôles (c.5), la motivation (c.6), le leadership (c.7), ainsi que le climat organisationnel (c.8). Dans chacun de ces domaines, les auteurs proposent effectivement des reformulations intéressantes.

A Theory of Behavior in Organizations est un livre assez difficile à lire. Il réclame du lecteur beaucoup d'attention et il faut une bonne dose de motivation pour tenir le coup tout au long des 299 pages que compte le volume. L'effort requis en vaut-il la peine? C'est difficile à dire. Une chose est à peu près certaine, cependant, et c'est que les futurs auteurs de théories et de modèles explicatifs du comportement humain dans les organisations devront, à l'avenir, tenir compte des positions exprimées dans ce livre par les auteurs Naylor, Pritchard et Ilgen. Les personnes intéressées à se situer à la fine pointe du développement des théories en ce domaine feront donc un investissement rentable en s'assurant d'avoir pris connaissance de ce modèle. Pour la majorité des gens, cependant, et pour les praticiens, il est peut-être préférable d'attendre la parution de versions plus digestibles ou plus pratiques (i.e. opérationnelles) de certains aspects de la présente théorie.

\section{André PETIT}

Université de Sherbrooke
Kent Rowley, The Organizer, A Canadian

Union Life, by Rick Salutin, Toronto, James Lorimer, 1980, 163 pp.

Kent Rowley n'est certes pas un nom bien connu dans l'histoire du Canada. Il n'était ni politicien ni homme d'affaires; il ne fut qu'un organisateur de travailleurs et travailleuses canadiens pendant quarante ans, bouleversant l'action ouvrière de la grande crise économique jusqu'à sa mort en 1978 . Pourquoi donc lui consacrer une biographie? C'était le plus grand leader syndical de son époque, selon l'auteur. Salutin essaie, dans ce petit livre de vulgarisation, de faire connaître en profondeur ce militant nationaliste et socialiste d'avant-garde, voire de corriger l'injure de ceux qui ont censuré les faits de la vie de Rowley. C'est le défi que l'auteur a cherché à relever.

Salutin trace chronologiquement la trame d'événements de la carrière de Rowley. Celui-ci est né à Montréal d'une famille bourgeoise. Néanmoins, il n'a pas eu l'occasion de poursuivre ses études après l'école secondaire. Au moment où il commence à travailler, il a été mêlé aux problèmes des ouvriers montréalais, en syndicalisant pour l'Office Employees International Union (FAT) les membres du personnel de son bureau. Un peu plus tard, il devenait l'organisateur à temps complet de l'United Textile Workers of America (FAT) dont il est devenu le secrétaire puis le président canadien, après avoir occupé divers postes exécutifs syndicaux. Dans son travail, il se heurtait, entre autres, à la CTCC, à la Dominion Textile, et surtout au premier ministre du Québec Maurice Duplessis. À la même période, il a fait la connaissance de sa proche collaboratrice pour les prochaines 35 années, Madeleine Parent, avec qui il se maria.

Malgré tout cela, ce n'était tout de même pas la vie en rose: Rowley a beaucoup souffert de diverses formes de répressions. Au début de la Deuxième guerre mondiale, il eu beau essayé de se faire soldat canadien, il fut libéré du service militaire. Soupçonné d'appartenir au parti communiste, le gouvernement l'a mis en prison pendant plus de deux ans. En 1946, c'était au tour de Duplessis de l'y mettre brièvement au cours de la grève de 
Valleyfield. L'accusation d'être communiste le suivit et durant la guerre froide où la chasse aux communistes et aux gauchistes prit son essor. La direction américaine de l'UTWA, sous la pression de Duplessis et du président de la Dominion Textile, Blair Gordon, prétexta cette excuse pour le licencier. Nul doute, ces agissements sournois et l'ingérence des chefs internationaux le poussaient vers le syndicalisme canadien nationaliste, ce qui est devenu la pierre angulaire de ses activités syndicales. Constamment harcelé par les syndicats catholiques, les syndicats internationaux, les patrons et le gouvernement Duplessis, il s'est rendu en Ontario où il fonda le Conseil canadien de textile à Hamilton qui n'a pas eu de succès. Inébranlable, il décida de promouvoir un congrès canadien - le Conseil des syndicats canadiens - plus tard appelé la Confédération des syndicats canadiens dont il devenait le secrétaire-trésorier. La CSC, en s'unissant aux syndicats nationalistes, ne devint pas un congrès puissant mais elle a cependant été la force motrice de la bataille nationaliste en attirant les "breakaways" comme Steel à Kitimat et le forum pour démontrer l'exploitation des canadiens par les internationaux. Rowley a maintenu avec acharnement jusqu'à sa mort la lutte pour se débarrasser du joug des syndicats internationaux.

L'auteur soulève, pas ouvertement, la dialectique de la lutte des classes vécue par Rowley et il aborde aussi un point capital: les difficultés de n'importe quel dissident qui interroge à fond l'exploitation des ouvrières et le système capitaliste. L'" "establishment" rejette la réalité des classes lorsqu'il nuit à ceux qui les critiquent en les accusant d'être communistes ou de quelque chose de semblable. En dépit de cela, Rowley ne se soumettait pas. Au contraire, il a sonné le tocsin pour un syndicalisme national en soulignant que les problèmes des ouvriers canadiens sont: soit les mauvaises conditions de travail ou les piètres salaires, soit l'entrave des syndicats internationaux. Il n'a donc pas gaspillé son temps à réfuter les accusations répandues d'être communiste, ou révolutionnaire. Il n'en était pas un, et pourtant, Salutin, au lieu de le constater tout simplement, aurait pu en fournir la preuve aux lecteurs, car il y en a.

De cette façon, parmi d'autres points fondamentaux, on sent l'amateurisme de cet exposé qui ne démontre aucun esprit critique. Sans aucun doute, Salutin est un admirateur de son héros, mais l'objectivité risque d'en souffrir et c'est ce qui est arrivé. En effet, il évite l'explication et l'interprétation; de plus, il ne se pose pas de questions. On peut, bien entendu, s'en tenir à la méthode d'histoire orale avec les louanges de Rowley mais il y a le revers de la médaille; il avait ses critiques et il aurait été préférable de les inclure pour pouvoir les détruire. Avec les manuscrits Rowley-Parent et d'autres qui sont disponibles, l'auteur aurait pu le faire facilement. Sans ces citations, certaines interprétations sont pour le moins incomplètes, et le lecteur ne peut qu'en imaginer les explications. Soulignons deux exemples parmi plusieurs: pourquoi Rowley a-t-il syndicalisé les internationaux pendant une quinzaine d'années en acceptant leur continentalisme jusqu'à ce qu'il soit congédié et devienne ensuite nationaliste? Comment explique-t-il la scission des Mine Mill Workers, un des syndicats fondateurs de la CSC? On peut masquer ces faits ou détourner l'attention du lecteur avec de longues incursions dans le temps et dans l'espace sans aucun but. En somme, il semble que Salutin n'ait pas fait de distinctions entre l'essentiel et le superflu.

Le caractère hagiographique de cette oeuvre quasi exclusivement descriptive n'est pas équitable pour Rowley non plus. Il a eu une vie pleine et il s'agit d'analyser sa vie et sa carrière, ce que Salutin n'a pas fait du tout. En raison de ces multiples lacunes, cet ouvrage est loin d'apporter le mot final sur cet homme extraordinaire; Rowley mérite mieux que ce que nous offre Salutin.

F.J.K. GRIEZIC

Carleton University 